\title{
(2) OPEN ACCESS \\ Imaging assessment of mitral and aortic regurgitation: current state of the art
}

\author{
Richard Paul Steeds [1] , ${ }^{1,2}$ Saul G Myerson (10 ${ }^{3}$
}

'Department of Cardiology, Queen Elizabeth Hospital, Birmingham, UK

${ }^{2}$ Honorary Reader, Institute of Cardiovascular Sciences, University of Birmingham, Birmingham, UK

${ }^{3}$ Cardiovascular Medicine, University of Oxford, Oxford, UK

\section{Correspondence to}

Dr Richard Paul Steeds, Department of Cardiology, Queen Elizabeth Hospital, Birmingham B15 2TH, UK rick.steeds@uhb.nhs.uk

Published Online First 17 August 2020

Check for updates

(C) Author(s) (or their employer(s)) 2020. Re-use permitted under CC BY. Published by BMJ.

To cite: Steeds RP Myerson SG. Heart 2020;106:1769-1776.

\section{INTRODUCTION}

Valvular heart disease (VHD) affects 1 in 50 of the general population and 1 in 3 of the over $65 \mathrm{~s}$, and is associated with increased morbidity and premature mortality. ${ }^{1}$ Among adults presenting to hospital with severe VHD, mitral regurgitation (MR: 21.3\%) is the second and aortic regurgitation (AR: $5.3 \%$ ) is the third most common valve lesion after aortic stenosis $(41.2 \%){ }^{2}$ The predominance of MR above AR is also found in community-based studies, although it is much more common to find mild and moderate VHDs than severe disease in the general population. ${ }^{1} \mathrm{VHD}$ of all types is more common with advancing age, and given the steady rise of life expectancy in the Western world, the frequency of patients presenting with MR and AR is increasing. Despite clear evidence of benefit from surgery when performed in a timely fashion, many patients undergo operation late in the course of disease, with more advanced symptoms and higher rates of heart failure and ventricular dysfunction. ${ }^{2}$ There is a long latent period in the majority of patients with either MR or AR, and timely diagnosis, careful monitoring and early referral remain critical to care.

\section{HAEMODYNAMICS AND IMAGING OF VALVULAR REGURGITATION}

The severity of regurgitation through the MV and AV is governed by the Gorlin formula, which states that flow through an orifice varies by the square root of the pressure gradient across the orifice, the duration of flow and a discharge coefficient. ${ }^{3}$ Therefore, the main determinants of the regurgitant volume (RVol) are (1) the size of the regurgitant orifice; (2) the pressure gradient between the left ventricle (LV) and the left atrium (LA) in MR, and between the aortic root and the LV in AR. In most patients, the size of the regurgitant orifice is not fixed but varies with dynamic changes in geometry, pressure and volume. For example, changes in volume status can have a significant impact on the assessment of MR, with reduction in severity following dialysis and after diuretic therapy compared with imaging assessment before treatment. Both the dynamic nature of the regurgitant orifice and the relationship between pressure and flow mean that images of the patient with regurgitation must be understood in the context of the haemodynamic status of the patient. Therefore, all reports of severity should include heart rate and blood pressure, while the use of sedation is an important consideration when comparing findings on transthoracic echocardiography (TTE) and transoesophageal echocardiography (TOE). Equally, it should be borne in mind

\section{Learning objectives}

- To understand the advantages and disadvantages of imaging techniques in identifying aetiology and mechanisms of mitral and aortic regurgitation.

- To understand the key echocardiographic methods of quantifying severity of mitral regurgitation (MR) and aortic regurgitation (AR), and their advantages and limitations.

- To be aware of the relative advantages and disadvantages of cardiac magnetic resonance in quantifying severity of MR and AR.

that most assessments of MR and AR occur at rest, and there may be significant changes on exertion (either an increase or decrease in MR, depending on the mechanism; and a decrease in AR due to the shortening of diastole with higher heart rates). Another important factor in assessing regurgitation is the time course of onset: for example, in chronic MR, there is chamber dilatation, increased LV compliance and lower pulmonary venous pressure, whereas in acute MR, which is usually into a low-compliance $\mathrm{LV}$, there is little cavity dilatation and high pulmonary venous pressure. In acute MR, this means the RVol may be much smaller than expected, and even a moderate degree may cause symptoms as the LV has not had time to accommodate for the MR. ${ }^{4}$

\section{IDENTIFYING THE MECHANISM OF PATHOLOGICAL REGURGITATION}

Access to echocardiography is critical for diagnosis of valve disease. ${ }^{5}$ TTE with colour Doppler remains the main modality for detection of regurgitation. Note that small degrees of physiological MR through a structurally normal valve will be detected in 10\%-20\% of children and closing volumes in a higher percentage. ${ }^{6}$ In contrast, physiological AR is rare, occurs in less than $1 \%$ of cases and should be considered pathological until proven otherwise.

Once regurgitation has been detected, the key step is to determine the cause. This is particularly the case in the patient with MR, as the natural history, classification of severity and treatment choices all depend on whether regurgitation is 'primary', due to a fault in the valve apparatus, or 'secondary', due to disease of the LV (table 1). Most cases of AR, on the other hand, are secondary to an aortic syndrome, either as a result of atherosclerotic aortic root dilatation or as part of the aortopathy that can coexist in 
Education in Heart

Table 1 Causes of mitral regurgitation

\begin{tabular}{|lll}
\hline Primary (valve apparatus) & Usual Carpentier \\
\hline Congenital & Cleft & mechanism \\
\hline Myxomatous & Parachute & II \\
\hline Degenerative & Fibroelastic deficiency & II \\
\hline Inflammatory & Barlow disease & II \\
\hline & Muc-related & \\
\hline & Rheumatic & IIIA \\
\hline Secondary (ventricular/atrial) & Radiation (lymphoma, breast) & IIIA \\
\hline Ischaemic & Drugs (dopamine agonists) & IIIA \\
\hline & Infective endocarditis & IB \\
\hline Non-ischaemic & Myocardial infarction & \\
\hline & LV dilatation/dysfunction & IIIB \\
\hline & LV dilatation/dysfunction & I \\
\hline
\end{tabular}

LV, left ventricle.

those with a bicuspid aortic valve ${ }^{7}$ (table 2 ). In both MR and AR, it is good practice to use the Carpentier classification to describe abnormalities of leaflet motion and thereby define mechanism (figures 1-5). This helps to identify the pathology, may guide the approach to surgery and can help to predict recurrence. ${ }^{8}$

Identifying structural abnormalities leading to MR requires imaging of the leaflets, chordae, papillary muscles and annulus at high temporal and spatial resolution. 2D TTE is the main method to define leaflet thickening (use fundamental imaging), leaflet mobility (normal, increased (including prolapse) or restricted), degree of leaflet coaptation, as well as extent and location of calcification (none, mild, moderate and severe). 3D TTE is of incremental value in identifying the lesion, leaflet involved and the specific

Table 2 Causes of aortic regurgitation

\begin{tabular}{lll}
\hline Primary (valve apparatus) & & \\
\hline Congenital & Bicuspid, quadricuspid & \\
\hline Myxomatous & Ventricular septal defect & \\
\hline Degenerative & Fibroelastic deficiency & IIIB \\
\hline Inflammatory & Age-related & IIIA \\
\hline & Mucopolysaccharidoses & IIIA \\
\hline & Rheumatic & IIIA \\
\hline Secondary (aortic) & Drugs (dopamine agonists) & IIIA \\
\hline Congenital & Infective endocarditis & IB \\
\hline \multirow{2}{*}{ Acquired } & Collagen vascular diseases & \\
\hline & Annuloaortic ectasia & I \\
\hline & Loezs-Dietz, Marfan, Ehlers-Danloss & I \\
\hline & Hypertension & I \\
\hline & Atherosclerotic & I \\
\hline
\end{tabular}

valve segments affected, with an accuracy similar to multiplane $2 \mathrm{D}$ TOE. ${ }^{9} 3 \mathrm{D}$ TOE has greatest accuracy overall compared with surgical findings, especially in identifying flail segments and the location and extent of prolapsed segments. While 2D TTE can diagnose the apical displacement of the mitral coaptation point, restriction in mobility and asymmetric tethering that are typical findings in ischaemic MR, 3D TTE and TOE quantify the extent of tethering that can direct surgical strategies with greater accuracy. ${ }^{10}$ Cardiovascular magnetic resonance (CMR) can also identify leaflet (including segmental) anatomy and motion using $2 \mathrm{D}$ cine imaging, although true $3 \mathrm{D}$ imaging is not available. It is best used when echocardiography is unable to image the valve adequately.

Visualisation of primary and secondary chordae requires good quality 2D TTE imaging in a modified apical two-chamber view to define location, length and thickening, with the transgastric longaxis view on 2D TOE also useful to perform these measurements and define interpapillary muscle distance. 3D TOE can improve on these measures but is mainly used to measure annulus size and detect annular remodelling that can be specific to a disease process, for example, to myxomatous or ischaemic MR. ${ }^{11}$ CMR can accurately locate, measure size and track papillary muscle displacement, but the slice thickness $(5-7 \mathrm{~mm})$ means that it is less useful for imaging the chordae. CMR can also identify associated fibrosis or infarction (in either the myocardium or papillary muscles), ${ }^{12}$ which can inform the assessment of the mechanism of MR and likelihood of improvement. Contrast-enhanced multislice CT can measure mitral annular size with similar accuracy and reproducibility to $3 \mathrm{D}$ TOE and has the advantage of accurate localisation and quantification of calcification. ${ }^{13}$ As a result, CT has a growing role in preprocedural imaging for percutaneous mitral intervention but does not yet play the central role it performs in assessment for transcatheter aortic intervention. It can also assess leaflet motion with full cardiac cycle imaging, although the temporal resolution is much lower than echocardiography or CMR, and the radiation dose is significantly higher, so this is generally reserved for rare situations when neither echocardiography nor CMR is able to provide functional information.

In the anatomical assessment of AR, 2D (and to some extent 3D) TTE remain the first step in the assessment of leaflet mobility, thickening and calcification required to determine mechanism and aetiology. When TTE provides inadequate visualisation of structures, or when high spatial and temporal resolution imaging is required to assess suitability for intervention, for example, with highly eccentric jets and when repair is considered, 2D and 3D TOEs provide incremental accuracy. ${ }^{14}$ Since many patients presenting with severe AR have aortic root or supracoronary ascending aortic enlargement, an additional requirement is for accurate multiplanar imaging of the aortic root and ascending aorta. Although TTE, TOE and CMR can offer 

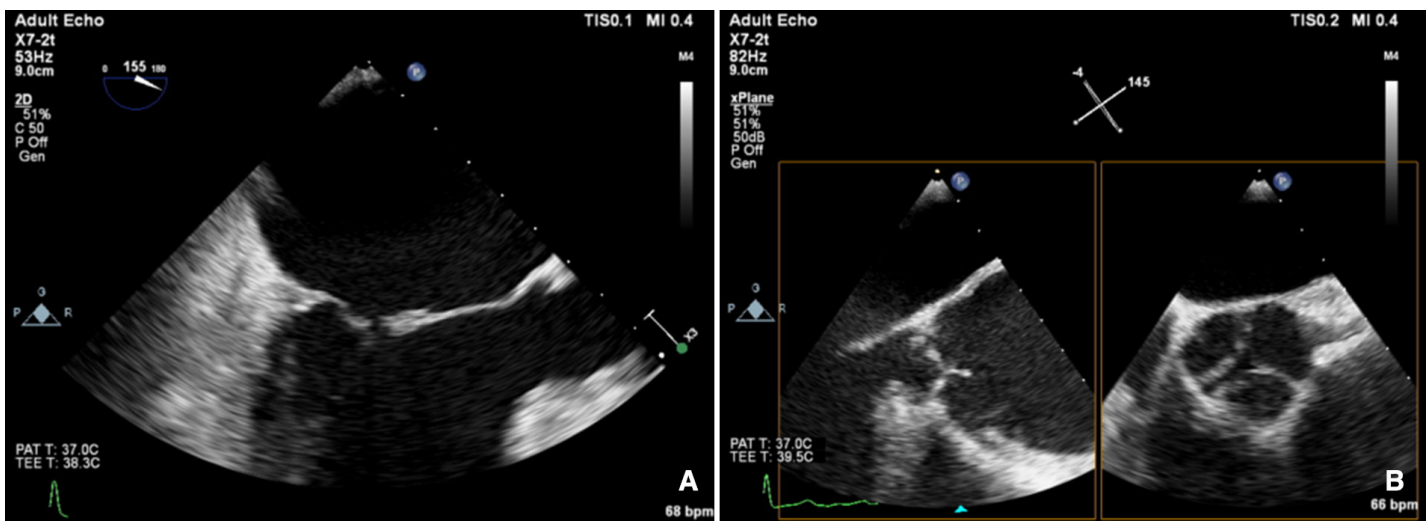

Figure 1 Carpentier type 1A, normal leaflet motion: annular dilatation $(A)$ mitral regurgitation and $(B)$ aortic regurgitation.

this, multidetector CT provides fast, high resolution imaging of the aortic root and thoracic aorta without limitation by acoustic window and is the technique of choice, not least due to the capacity to locate and quantify calcification. ${ }^{15}$

\section{SEVERITY OF REGURGITATION}

When quantifying severity of a regurgitant lesion, it is important to consider the appearance of (1) the valve morphology and (2) the size, shape and function of the ventricle. For example, if the mitral valve leaflets are flail, it is highly likely there is severe regurgitation but if you do not think it is severe, make sure you have done all you can to be certain. Likewise, if the LV is dilated with spherical remodelling, if you are going to call any AR moderate, make sure you have done all you can to be certain.

Good quality TTE with Doppler can provide all the core information required for evaluation of regurgitation severity but requires an experienced, motivated echocardiographer able to perform the necessary integrated assessment of multiple qualitative and quantitative parameters, in the knowledge that single measures are subject to variability ${ }^{16}$ (table 3). A practical problem is that there is overreliance on colour Doppler, particularly if limited to visualisation of the regurgitant jet area, which is inaccurate (even if indexed to chamber area). ${ }^{17}$ Despite guidelines recommending that regurgitation is quantified using methods including vena contracta and flow convergence on colour Doppler, many decisions regarding severity of MR are still made on echo by visually estimating severity based on the size of the colour jet, with some including semiquantitative measures (vena contracta width, regurgitant orifice area), but only a minority of reports provide RVols and fraction. ${ }^{18}$ Flow convergence, better known as the proximal isovelocity surface area (PISA) method, is probably the most widely used method used to quantify MR, which relies on measuring the radius of a hemisphere from the point of aliasing on colour Doppler to the vena contracta of the jet. The effective regurgitant orifice (EROA) is then calculated from the maximal velocity of the regurgitant jet, and the RVol by multiplying this with the velocity time integral of the MR jet. ${ }^{19}$ Although quantitative grading of $\mathrm{MR}$ is an important independent imaging marker of outcome, ${ }^{20}$ it involves a number of assumptions and calculation from multiple measurements, with significant variation between expert readers, and there is increasing awareness of inaccuracy where the MR does not form a true hemisphere, particularly in functional regurgitation. ${ }^{21}$

The assessment of AR severity with echocardiography is more challenging than that of MR. On TTE, the AR jet is seen with the highest resolution in the parasternal long-axis view, which can be used to measure vena contracta and jet width relative to the left ventricular outflow tract. In this
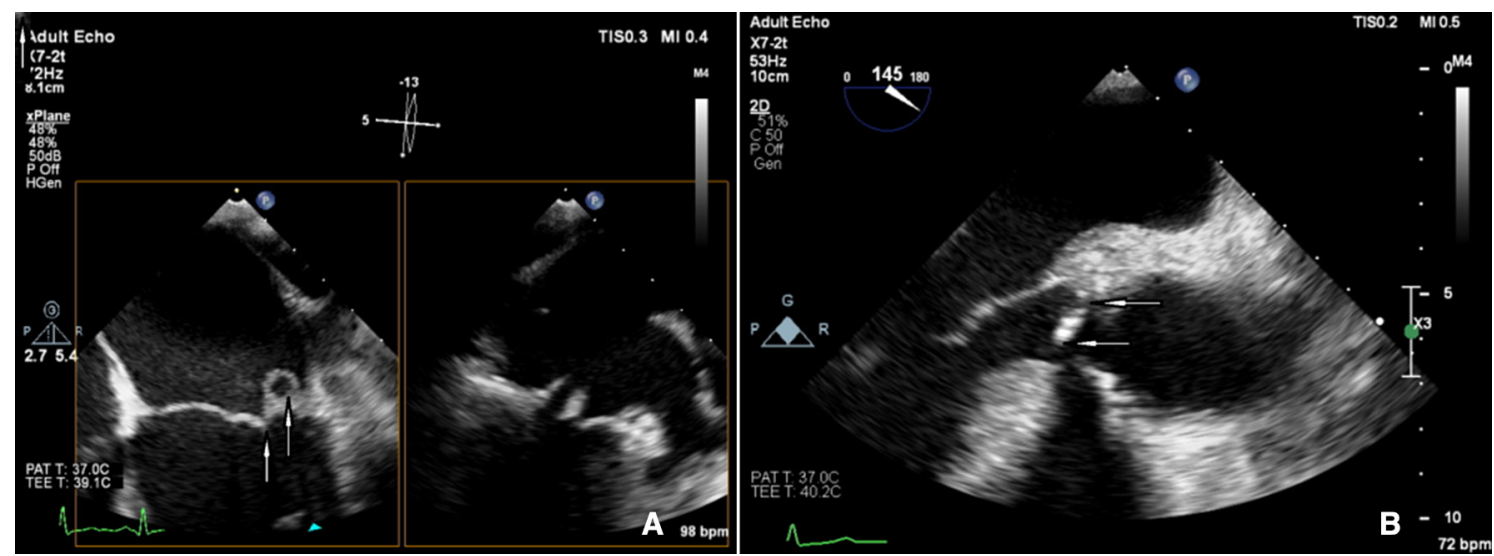

Figure 2 Carpentier type 1B, normal leaflet motion: leaflet perforation (A) mitral regurgitation and (B) aortic regurgitation. 


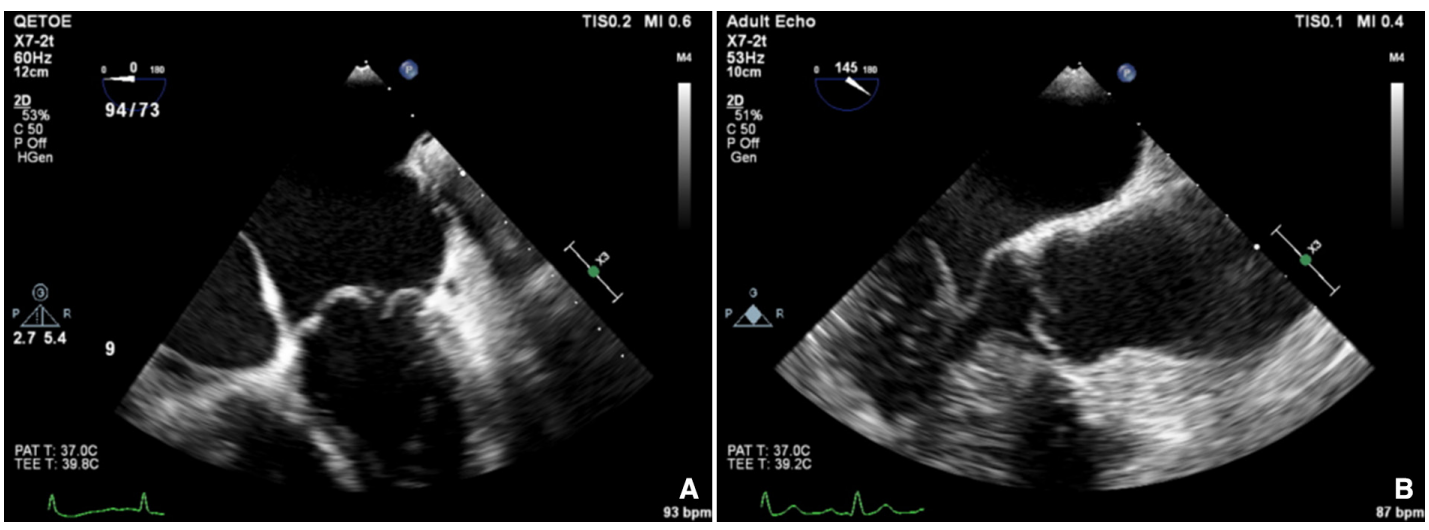

Figure 3 Carpentier type 2: excessive leaflet motion with prolapse of leaflet tip behind annulus (A) mitral regurgitation and (B) aortic regurgitation.
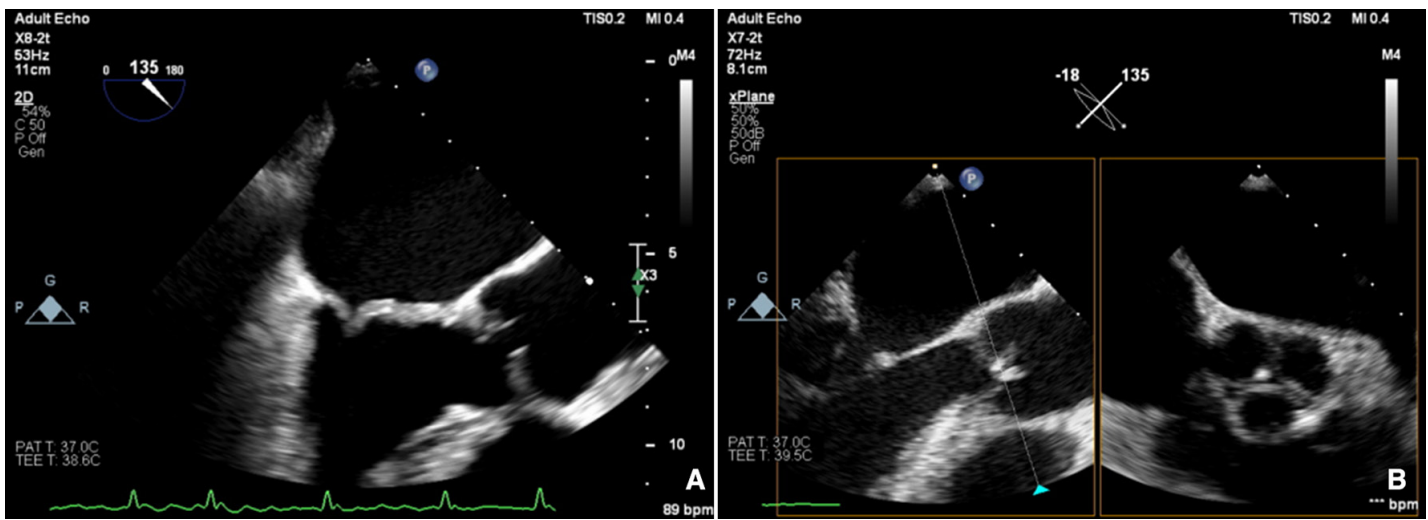

Figure 4 Carpentier type 3A: restrictive leaflet motion—systole and diastole: rheumatic (A) mitral regurgitation and (B) aortic regurgitation.

view however, the PISA in AR is perpendicular to the angle of insonation (and therefore unreliable), and in the apical window it is in the far field and is often too small to measure reliably. In addition, the pulse Doppler method used to calculate stroke volumes across the mitral valve and left ventricular outflow tract ( $\mathrm{AR} \mathrm{RVol}=\mathrm{SVol}_{\mathrm{LVOT}}-\mathrm{SVol}$ мұ) is subject to large interobserver variability. ${ }^{2}$ Other methods such as pressure half time of the AR Doppler slope can be helpful to identify mild or severe disease but may be unreliable when there are changes in LV compliance or use of

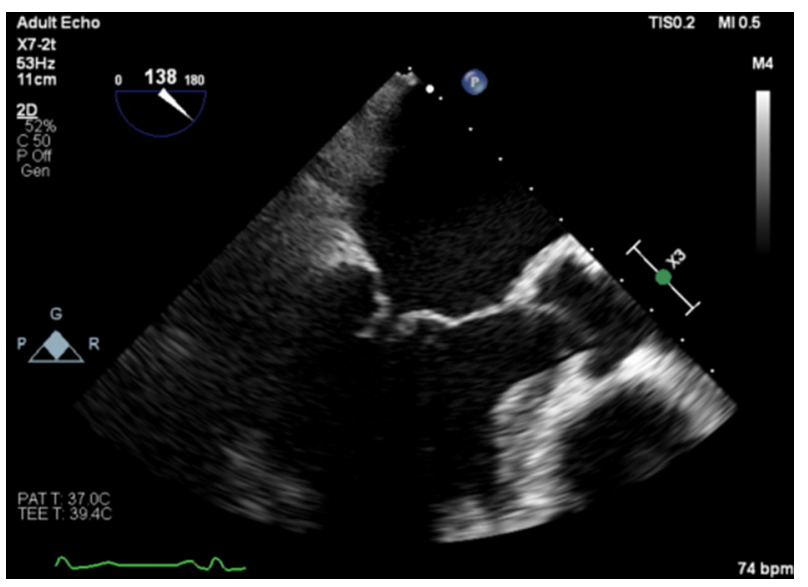

Figure 5 Carpentier type 3B: restrictive leaflet motion—systole (closure): ischaemic mitral regurgitation. antihypertensive medication, and likewise, aortic diastolic flow reversal in the descending aorta may be useful but can also occur due to changes in aortic distensibility in the elderly.

There is increasing recognition of the limitations of echocardiography in quantifying regurgitation, particularly in differentiating moderate from severe regurgitation. ${ }^{23} \mathrm{CMR}$ has the advantage of direct flow quantification using phase contrast velocity mapping. When used in the aortic root, this can quantify AR Rvol directly and can also be used to quantify MR indirectly, in conjunction with LV stroke volumes (MR RVol=LV stroke volumeaortic forward flow). This has greater reproducibility than multiparametric echocardiography for RVol and grade ${ }^{24}$ and has good accuracy in in vitro models. ${ }^{25}$ Echo tends to overestimate severity compared with CMR, especially when MR varies throughout systole, ${ }^{26}$ and CMR is a better predictor of the future requirement for surgery, and ventricular remodelling postsurgery in the limited number of studies to date. ${ }^{27}$ Cut-offs that predict adverse events or need for intervention are therefore lower for both $\mathrm{MR}^{28}$ and $\mathrm{AR},{ }^{29}$ and are not always the same as guidelines based on echo parameters (see table 3). CMR is not without problems, including background phase offset error, variation in volumes due to difference in selection of the basal slice and impact of irregular heart rhythms, and is not as widely available as echocardiography. CMR is most useful for sorting out difficult cases, such as 
Table 3 Multiparametric assessment of regurgitation

2D colour Doppler jet area

$\begin{array}{ll}\text { Advantages } & \text { Limitations } \\ \text { Easy screening for mild or } & \text { Subjective } \\ \text { severe } & \text { Overestimates if transi } \\ \text { If narrow, specific for mild } & \text { Underestimates if } \\ & \text { Wall hugging. } \\ & \text { Eccentric. } \\ & \text { Variable a/c to } \\ & \text { Machine settings. } \\ & \end{array}$

2D colour Doppler vena contracta width

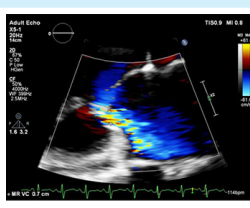

2D colour Doppler flow convergence

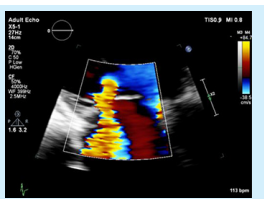

3D colour Doppler vena contracta area

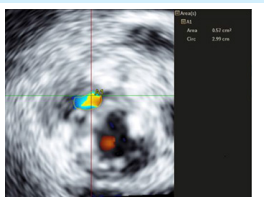

CW Doppler density

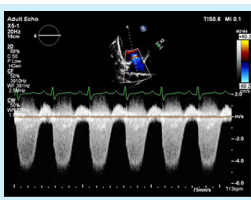

2D colour Doppler PISA

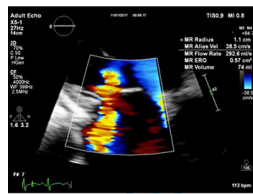

Pulse Doppler

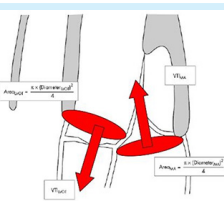

CMR flow quantification

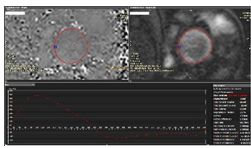

Easy

Dense triangular shape specific for severe MR

Good for mild or severe Independent of flow and pressure

Useful in eccentric jets Marker for ROA

Easy
If absent, specific for mild

Multiple jets

Multiple jets

Overestimates if transient

$<3 \mathrm{~mm}$

$\mathrm{MR}>7 \mathrm{~mm}$

Must be measured when US is perpendicular

Overestimates if transient.

- Non-hemispheric.

Underestimates if

- Wall hugging.

- Eccentric.

Useful if:

Multiple jets

If PISA non-hemispheric

Limits of temporal and spatial

If no flow convergence $>10 \mathrm{~mm}$ if Nyquist $30-40 \mathrm{~cm} / \mathrm{s}$

can be seen

$A R>6 \mathrm{~mm}$

Wide origin, large MR $>50 \%$ MR/LA area $A R>65 \%$ jet width/LVOT

Narrow origin, small

Quantitative:

EROA mm2

RVol mls

RF \%

Prognostic

Quantitative:

RVol mL

RF \%

Can be used in

Eccentric

Transient

Multiple jets

Accurate quantification

of flow

Not affected by irregular, eccentric, multiple or dynamic jets

Easy to measure

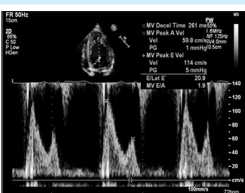

Easy

Quantitative resolution $3 \mathrm{D}$ CF

Overestimates if transient Slow

Subjective

Gain dependent

Underestimates eccentric

Feint

Altered by LA/LV pressure

A wave dominant Specific for mild MR

MR EROA $<20 \mathrm{~mm}$ AR EROA $<10 \mathrm{~mm}$ $\mathrm{RVol}<30 \mathrm{~mL}$

Transient

$\mathrm{RF}<30 \%$

Wide confidence limits

Difficult

Not useful if multiple valve

Primary MR RVol

$<30 \mathrm{~mL}$

MR RF $<30 \%$

AR RVol $<30 \mathrm{~mL}$

AR RF $<30 \%$

Accurate measures often require $\mathrm{MR} \mathrm{Vol}<30 \mathrm{~mL}$ good correction of background MR RF $<20 \%$ flow offset error

AR RF $<15 \%$

Slight tendency for AR quantities

to be underestimated

Not available in all hospitals

$>40 \mathrm{~mm} 2$

Dense

SIMR $>20 \mathrm{~mm}$

AR EROA $>30 \mathrm{~mm}$

RVol $>60 \mathrm{~mL}$

$\mathrm{RF}>50 \%$

Primary MR RVol $>60 \mathrm{~mL}$

MR RF $>50 \%$

Secondary MR RVol $>30 \mathrm{~mL}$

AR RVol $>60 \mathrm{~mL}$

AR RF $>50 \%$

MR Vol $>60 \mathrm{~mL}$

MR RF $>40 \%$

AR RF $>35 \%-40 \%$

Holodiastolic descending aorta

flow reversal

$\mathrm{EV} \max >1.5 \mathrm{~m} / \mathrm{s}$ gradient

AF

$<1$

$>1.4$

Not useful if multiple valve

disease 


\begin{tabular}{|c|c|c|c|c|}
\hline Parameter & Advantages & Limitations & Mild & Severe \\
\hline $\begin{array}{l}\text { Pulse Doppler pulmonary } \\
\text { venous flow }\end{array}$ & $\begin{array}{l}\text { Easy on TOE } \\
\text { Useful in eccentric or } \\
\text { multiple jets }\end{array}$ & $\begin{array}{l}\text { Systolic blunting occurs if high } \\
\text { LA or LV end-diastolic pressure } \\
\text { Other causes for high E Vmax }\end{array}$ & Systolic flow dominant & Systolic flow reversal \\
\hline \multicolumn{5}{|l|}{ AR only } \\
\hline $\begin{array}{l}\text { Pulse Doppler descending } \\
\text { aortic flow }\end{array}$ & $\begin{array}{l}\text { Easy } \\
\text { If present in abdominal } \\
\text { aorta, specific for severe } \\
\text { AR }\end{array}$ & $\begin{array}{l}\text { Less reliable when aortic } \\
\text { stiffness increased (age) } \\
\text { Depends on alignment of } \\
\text { Doppler with jet direction } \\
\text { Variable according to location } \\
\text { of sample }\end{array}$ & $\begin{array}{l}\text { Brief flow reversal is } \\
\text { normal }\end{array}$ & $\begin{array}{l}\text { Holodiastolic, end-diastolic } \\
\text { flow Vmax }>20 \mathrm{~cm} / \mathrm{s}\end{array}$ \\
\hline $\begin{array}{l}\text { CW Doppler pressure } \\
\text { half-time }\end{array}$ & $\begin{array}{l}\text { Easy } \\
\text { If long, specific for mild }\end{array}$ & $\begin{array}{l}\text { Affected by any factor altering } \\
\text { aortic-LV pressure gradient } \\
\text { Depends on alignment of } \\
\text { Doppler with jet direction }\end{array}$ & $>500 \mathrm{~ms}$ & $<200 \mathrm{~ms}$ \\
\hline
\end{tabular}

a/c, according to; AR, aortic regurgitation; CF, colour flow; CMR, cardiovascular magnetic resonance; EROA, effective regurgitant orifice; LA, left atrium; LV, left ventricle; LVOT, left ventricular outflow tract; MR, mitral regurgitation; MV, mitral valve; PISA, proximal isovelocity surface area; RF, regurgitant fraction; RVol, regurgitant volume; TOE, transoesophageal echocardiography; US, ultrasound.

'moderate-severe MR', discrepancy between symptoms and regurgitation grade, exercise capacity and severity of regurgitation on echo, and where TTE images are suboptimal.

\section{ASSESSING THE CONSEQUENCES OF REGURGITATION}

Chronic AR is characterised by combined volume and pressure overload. The LV increases diastolic volume to accommodate AR, which results in increased diastolic wall stress. As the LV must eject a greater stroke volume over the same systolic ejection period, systolic pressure, afterload and systolic wall stress progressively increase. The combination of volume and pressure overload result in LV dilatation, eccentric LV hypertrophy, and increased interstitial fibrosis, which are progressive with increasing severity of AR and can lead ultimately to heart failure and death. ${ }^{30}$ In chronic MR, volume overload produces similar LV changes, with gradual transition from a compensated phase, transitioning to LV impairment and ultimately decompensation. ${ }^{31}$ There is some CMR evidence that AR results in higher LV volumes for the same quantity of regurgitation, suggesting a differential LV response. ${ }^{32}$ Current guidelines emphasise the role of tracking LV chamber size and function, with cut-offs for intervention in asymptomatic patients based on linear dimensions and ejection fraction on $\mathrm{TTE}^{33}$ (tables 3 ). There are obvious limitations to using a single linear dimension as a trigger for intervention, not least that in primary MR, there is preferential remodelling of the LV in the apex and mid cavity. ${ }^{34}$ Volumetric data have not however, been established in guidelines as a trigger, whether by contrast TTE, 3D TTE or CMR, despite the greater reproducibility and accuracy in tracking change. Likewise, there are limitations to the use of ejection fraction in regurgitation, which measures chamber size rather than myocardial contractility and is altered by the RVol itself.

Multiple alternatives have been proposed for early detection of myocardial dysfunction, including fall in systolic and early myocardial relaxation velocities on tissue Doppler, reduction in strain, contractile reserve on stress echocardiography, and fibrosis on CMR, none of which have been accepted into guidelines or been subjected to randomised trial end-point adjudication. ${ }^{35}$

Measuring left atrial volume, RV size and function, and maximal velocity of the regurgitant TR jet are all important parts of the imaging assessment of the patient with MR that carry prognostic weight, and provide supportive evidence for a decision to intervene. ${ }^{16}$ It is important to recognise the limitations in accuracy of the calculation of pulmonary artery systolic pressure by echocardiography however, and the need for confirmation of the estimation of pulmonary pressure by invasive testing. ${ }^{36} \mathrm{CMR}$ offers greater inter-study reproducibility for volume measures of LV, RV and LA chamber size and ejection fraction, compared even to 3D TTE with or without contrast but proof of an advantage in routine assessment of the asymptomatic patient with MR or AR is lacking. ${ }^{37}$

\section{SUMMARY}

Imaging assessment of the patient with MR or AR requires detailed assessment of the valve apparatus, associated cardiac chambers, the aortic root (for $\mathrm{AR}$ ) and flow quantification (for CMR) to identify the cause and mechanism of regurgitation, severity, and haemodynamic impact on ventricular and atrial remodelling, as well as the effect on pulmonary pressure. Transthoracic echocardiography remains the workhorse but requires careful integration of multiple parameters that vary in sensitivity and specificity, particularly when the 
All imaging assessments in mitral or aortic regurgitation should include a statement on the quality of the scan, the size of the patient (height, weight and body surface area) and haemodynamic status (heart rate, blood pressure and procedural medication).

- Transthoracic echocardiography remains the first-line imaging modality to determine cause and mechanism of regurgitation, quantify severity using multiple parameters, and impact of regurgitation on atrial and ventricular size and function.

- In cases where the above information is incomplete or is contradictory, transoesophageal echocardiography using $2 \mathrm{D}$ and $3 \mathrm{D}$ is the usual next step for detailed assessment of valve anatomy and helps with quantification.

- In those cases where regurgitation severity and the effects of regurgitation on chamber size and function are the primary source of concern, cardiovascular magnetic resonance (CMR) may be a better, less invasive alternative.

- Grading of severity by echocardiography and CMR differ, with thresholds for intervention lower using CMR, although there is a lack of data using these to guide intervention.

- Multislice CT is currently used mainly to image specific subsets of patients before intervention, such as those for percutaneous valve intervention and those requiring concomitant aortic surgery. access article distributed in accordance with the Creative Commons Attribution 4.0 Unported (CC BY 4.0) license, which permits others to copy, redistribute, remix, transform and build upon this work for any purpose, provided the original work is properly cited, a link to the licence is given, and indication of whether changes were made. See: https:// creativecommons.org/licenses/ by $/ 4.0 \%$.

\section{ORCID iDs}

Richard Paul Steeds http://orcid. org/0000-0001-5687-2535 Saul G Myerson http://orcid.org/ 0000-0001-5927-0230

\section{REFERENCES}

$\begin{array}{cc}1 & \text { d'Arcy JL, Coffey } \\ \text { S, Loudon MA, et al. Large-Scale }\end{array}$ community echocardiographic screening reveals a major burden of undiagnosed valvular heart disease in older people: the OxVALVE population cohort study. Eur Heart J 2016;37:3515-22.

*2 lung $B$,

Delgado V, Rosenhek $R$, et al. Contemporary presentation and management of valvular heart disease: the EURObservational research programme valvular heart disease II survey. Circulation 2019. doi:10.1161/ CIRCULATIONAHA. 119.041080

[Epub ahead of print: $12 \mathrm{Sep}$ 2019].

3 Gorlin R, Gorlin SG Hydraulic formula for calculation of the area of the stenotic mitral valve, other cardiac valves, and

aetiology is ischaemic, and the skill to use $3 \mathrm{D}$ regularly. Where data are conflicting, imaging is suboptimal or when intervention is under consideration, either transoesophageal echocardiography or CMR can be used, with $3 \mathrm{D}$ an integral component for complete TOE assessment.

Twitter Richard Paul Steeds @RichardSteeds

Contributors Both authors contributed to the writing of this article.

Funding SGM is supported by the NIHR Oxford Biomedical Research Centre. The authors acknowledge the contribution and funding provided by the British Heart Foundation Accelerator Award AA/18/2/34218 to the Institute of Cardiovascular Sciences, University of Birmingham.

Competing interests None declared.

Patient and public involvement Patients and/or the public were not involved in the design, conduct, reporting or dissemination plans of this research.

Patient consent for publication Not required.

Provenance and peer review Not commissioned; externally peer reviewed.

Data availability statement There are no data in this work Author note References which include $a$ * are considered to be key references. central circulatory shunts. I. Am Heart J 1951;41:1-29.

4 Gaasch WH, Meyer TE. Left ventricular response to mitral regurgitation. Circulation 2008;118:2298-303.

5 Gardezi SKM, Myerson SG, Chambers J, et al. Cardiac auscultation asymptomatic primary care patients. Heart 2018;104:1832-5.

6 Webb RH, Gentles TL, Stirling JW, et al. Valvular regurgitation using portable echocardiography in a healthy student population: implications for rheumatic heart disease screening. J Am Soc Echocardiogr 2015;28:981-8.

7 Mentias A, Feng K, Alashi A, et al. Long-Term Outcomes in Patients With Aortic Regurgitation and Preserved Left Ventricular Ejection Fraction. J Am Coll Cardiol 2016;68:2144-53.

8 Boodhwani M, de Kerchove L, Glineur D, et al. Repair-oriented classification of aortic insufficiency: impact on surgical techniques and clinical outcomes. J Thorac Cardiovasc Surg 2009;137:286-94

9 Liu B, Edwards NC, Pennell D, et al. The evolving role of cardiac magnetic resonance in primary mitral regurgitation: ready for prime time? Eur Heart J Cardiovasc Imaging 2019;20:123-30.

10 Garbi M, Monaghan MJ. Quantitative mitral valve anatomy and pathology. Echo Res Pract 2015;2:R63-72.

11 Grewal J, Suri R, Mankad S, et al. Mitral annular dynamics in myxomatous valve disease: new insights with realtime 3-dimensional echocardiography. Circulation 2010;121:1423-31.

12 Han Y, Peters DC, Kissinger KV, et al. Evaluation of papillary muscle function using cardiovascular magnetic resonance imaging in mitral valve prolapse. Am J Cardiol 2010;106:243-8.

13 Mak GJ, Blanke P, Ong K, et al. Three-Dimensional echocardiography compared with computed tomography to poorly predicts the presence of valvular heart disease in 
determine mitral annulus size before transcatheter mitral valve implantation. Circ Cardiovasc Imaging 2016;9:e004176.

14 le Polain de Waroux J-B, Pouleur A-C, Goffinet C, et al. Functional anatomy of aortic regurgitation: accuracy, prediction of surgical repairability, and outcome implications of transesophageal echocardiography. Circulation 2007;116:I-264-I-269.

15 Goldstein SA, Evangelista A, Abbara S, et al. Multimodality imaging of diseases of the thoracic aorta in adults: from the American Society of echocardiography and the European association of cardiovascular imaging: endorsed by the Society of cardiovascular computed tomography and Society for cardiovascular magnetic resonance. J Am Soc Echocardiogr 2015;28:119-82.

*16 Zoghbi WA, Adams D, Bonow RO, et al. Recommendations for noninvasive evaluation of native valvular regurgitation: a report from the American Society of echocardiography developed in collaboration with the Society for cardiovascular magnetic resonance. J Am Soc Echocardiogr 2017:30:303-71.

17 Thomas JD, Liu CM, Flachskampf FA, et al. Quantification of jet flow by momentum analysis. An in vitro color Doppler flow study. Circulation 1990;81:247-59.

18 Wang A, Grayburn P, Foster JA, et al. Practice gaps in the care of mitral valve regurgitation: insights from the American College of cardiology mitral regurgitation gap analysis and Advisory panel. Am Heart J 2016;172:70-9.

19 Vandervoort PM, Rivera JM, Mele D, et al. Application of color Doppler flow mapping to calculate effective regurgitant orifice area. An in vitro study and initial clinical observations. Circulation 1993:88:1150-6.

*20 Enriquez-Sarano M, Avierinos J-F, Messika-Zeitoun D, et al. Quantitative determinants of the outcome of asymptomatic mitral regurgitation. N Engl J Med 2005;352:875-83.

*21 Biner S, Rafique A, Rafii F, et al. Reproducibility of proximal isovelocity surface area, vena contracta, and regurgitant jet area for assessment of mitral regurgitation severity. JACC CardiovasC Imaging 2010;3:235-43.

22 Thavendiranathan P, Liu S, Datta S, et al. Automated quantification of mitral inflow and aortic outflow stroke volumes by threedimensional real-time volume color-flow Doppler transthoracic echocardiography: comparison with pulsed-wave Doppler and cardiac magnetic resonance imaging. J Am Soc Echocardiogr 2012:25:56-65

23 Uretsky S, Gillam L, Lang R, et al. Discordance between echocardiography and MRI in the assessment of mitral regurgitation severity: a prospective multicenter trial. J Am Coll Cardiol 2015;65:1078-88

*24 Penicka M, Vecera J, Mirica DC, et al. Prognostic implications of magnetic Resonance-Derived quantification in asymptomatic patients with organic mitral regurgitation: comparison with Doppler Echocardiography-Derived integrative approach. Circulation 2018;137:1349-60.
25 Chatzimavroudis GP, Oshinski JN, Franch RH, et al. Evaluation of the precision of magnetic resonance phase velocity mapping for blood flow measurements. J Cardiovasc Magn Reson 2001;3:11-19.

26 Uretsky S, Aldaia L, Marcoff L, et al. The Effect of Systolic Variation of Mitral Regurgitation on Discordance Between Noninvasive Imaging Modalities. JACC Cardiovasc Imaging 2019;12:2431-42.

27 Aplin M, Kyhl K, Bjerre J, et al. Cardiac remodelling and function with primary mitral valve insufficiency studied by magnetic resonance imaging. Eur Heart J Cardiovasc Imaging 2016:17:863-70.

28 Myerson SG, d'Arcy J, Christiansen JP, et al. Determination of clinical outcome in mitral regurgitation with cardiovascular magnetic resonance quantification. Circulation 2016;133:2287-96.

29 Myerson SG, d'Arcy J, Mohiaddin R, et al. Aortic regurgitation quantification using cardiovascular magnetic resonance: association with clinical outcome. Circulation 2012;126:1452-60.

30 Hess OM, Ritter M, Schneider J, et al. Diastolic stiffness and myocardial structure in aortic valve disease before and after valve replacement. Circulation 1984;69:855-65.

31 Gaasch WH, Zile MR. Left ventricular structural remodeling in health and disease. J Am Coll Cardiol 2011;58:1733-40.

32 Uretsky S, Supariwala A, Nidadovolu P, et al. Quantification of left ventricular remodeling in response to isolated aortic or mitral regurgitation. J Cardiovasc Magn Reson 2010;12:32

*33 Baumgartner H, Falk V, Bax JJ, et al. 2017 ESC/EACTS guidelines for the management of valvular heart disease. Eur Heart $J$ 2017;38:2739-91.

34 Matsumura T, Ohtaki E, Tanaka K, et al. Echocardiographic prediction of left ventricular dysfunction after mitral valve repair for mitral regurgitation as an indicator to decide the optimal timing of repair. J Am Coll Cardiol 2003;42:458-63.

35 Lee JKT, Franzone A, Lanz J, et al. Early detection of subclinical myocardial damage in chronic aortic regurgitation and strategies for timely treatment of asymptomatic patients. Circulation 2018;137:184-96.

36 Galiè N, Humbert M, Vachiery J-L, et al. 2015 ESC/ERS guidelines for the diagnosis and treatment of pulmonary hypertension: the joint Task force for the diagnosis and treatment of pulmonary hypertension of the European Society of cardiology (ESC) and the European respiratory Society (ERS): endorsed by: association for European paediatric and congenital cardiology (AEPC), International Society for heart and lung transplantation (ISHLT). Eur Heart J 2016;37:67-119.

37 Jenkins C, Moir S, Chan J, et al. Left ventricular volume measurement with echocardiography: a comparison of left ventricular opacification, three-dimensional echocardiography, or both with magnetic resonance imaging. Eur Heart $J$ 2009:30:98-106. 